\title{
Eye Position Representation in Human Anterior Parietal Cortex
}

\author{
Daniela Balslev ${ }^{1,2}$ and R. Chris Miall ${ }^{1}$ \\ ${ }^{1}$ School of Psychology, University of Birmingham, Edgbaston, Birmingham B15 2TT, United Kingdom, and 2Danish Research Centre for Magnetic \\ Resonance, Copenhagen University Hospital, DK-2650 Hvidovre, Denmark
}

\begin{abstract}
Eye position helps locate visual targets relative to one's own body and modulates the distribution of attention in visual space. Whereas in the monkey, proprioceptive eye position signals have been recorded in the somatosensory cortex, in humans, no brain site has yet been associated with eye position. We aimed to disrupt the proprioceptive representation of the right eye in the left somatosensory cortex, presumably located near the representation of the right hand, using repetitive transcranial magnetic stimulation (rTMS). Head-fixed subjects reported their perceived visual straight-ahead position using both left and right eye monocular vision, before and after 15 min of $1 \mathrm{~Hz}$ rTMS. rTMS over left somatosensory but not over left motor cortex shifted the perceived visual straight ahead to the left, whereas nonvisual detection of body midline was unchanged for either brain area. These results can be explained by the underestimation of the angle of gaze of the right eye when fixating the target. To link this effect more tightly to an altered ocular proprioception, we applied a passive deviation to the right eye before the visual straight-ahead task. Passive eye displacement modulated the shift in the perceived straight ahead induced by somatosensory rTMS, without affecting the perceived straight ahead at baseline or after motor cortex rTMS. We conclude that the anterior parietal cortex in humans encodes eye position and that this signal has a proprioceptive component.
\end{abstract}

Key words: eye; proprioception; visual; somatosensory; TMS; parietal cortex

\section{Introduction}

Information about eye position links the projection of a visual object on the retina to a location in space around the body. Eye position is taken into consideration when directing hand movements to visual targets (Gauthier et al., 1990) or when allocating attention in space (Craighero et al., 2004; Pavani et al., 2005).

Both eye muscle proprioception and the efferent copy of motor commands provide eye position information. The human extraocular muscles (EOMs) have muscle spindles that sense muscle length (Donaldson, 2000), so eye position may be computed by integrating the efferent signals with afferents from these sensors. Complete surgical deafferentation of the EOMs in the monkey leaves visual localization unimpaired, suggesting that in the absence of proprioception, the efference copy of eye movements is sufficient for visual localization (Lewis et al., 1998). However, although eye proprioception is not critical for visual localization, changes in proprioceptive input after passive eye displacement or after vibration of the extraocular muscles at a frequency known to stimulate the muscle spindles can produce changes in perceived location of visual targets relative to the body and in the accuracy of reaching (Gauthier et al., 1990; Han and Lennerstrand, 1999), supporting the idea that in healthy individ-

\footnotetext{
Received April 8, 2008; revised July 2, 2008; accepted July 23, 2008.

This work was supported by a postdoctoral fellowship from the Danish Medical Research Councils (D.B.). We thank Jonathan Winter for technical assistance.

This article is freely available online through the J Neurosci Open Choice option.

Correspondence should be addressed to Daniela Balslev, School of Psychology, University of Birmingham, Edgbaston, Birmingham B15 2TT, UK. E-mail: d.balslev@bham.ac.uk.

DOI:10.1523/JNEUROSCI.1513-08.2008

Copyright $\odot 2008$ Society for Neuroscience $\quad$ 0270-6474/08/288968-05\$15.00/0
}

uals visual localization not only relies on the efferent copy of the motor commands but also takes into consideration eye proprioception.

In the monkey, the position of the eyes in the head modulates the neural response to visual targets in the posterior parietal cortex (Andersen and Mountcastle, 1983), and specifically, a proprioceptive eye position signal has recently been recorded from the somatosensory cortex (Wang et al., 2007). In humans, however, no brain site has yet been associated with coding eye position.

To find out whether the somatosensory cortex in humans receives an eye position signal, we used $1 \mathrm{~Hz}$ repetitive transcranial magnetic stimulation (rTMS) to modulate somatosensory excitability over a potential cortical target. We placed the TMS coil over an anterior parietal area where TMS has previously been shown to alter hand proprioception (Balslev et al., 2004). Because of the proximity of hand and face representations in human somatosensory cortex [e.g., $<12 \mathrm{~mm}$ distance between thumb and forehead (DaSilva et al., 2002; Nguyen et al., 2004)] and because the area underneath the coil where TMS is effective is relatively large (Barker, 1999), we assumed that this somatosensory area may be a good candidate in the search for a proprioceptive representation of the EOMs. We show that somatosensory but not motor cortex rTMS increased the error in localization of visually perceived straight ahead and that brief passive eye displacement before the task modulated this effect (experiment 1 ). To rule out a potential effect of rTMS on the motor representation of the extraocular muscles as an explanation of our findings, we used the primary motor cortex as a control site. The location of the control site, anterior to the area of interest and hence closer to the eye, also rules out any direct effect of TMS on the eye muscles. 
Two control experiments in which the participants indicated their body midline by pointing with the head (experiment 2 ) or with their left index finger (experiment 3 ) ruled out an alternative hypothesis that the rTMS-induced error in visual localization relative to the body merely reflected an altered somatosensory body schema.

\section{Materials and Methods \\ Subjects}

All participants were healthy, right-handed adults with normal vision who gave written informed consent to participate in the study. The study was approved by the School of Psychology Ethics Committee at the University of Birmingham. For experiment 1 , we recruited eight participants (age median, 20; range, 18-23; 6 female, 5 right eye dominant). For experiment 2, we used 10 participants (age median, 22; range, 19-24; 8 female), and for experiment 3 , another 10 participants (age median, 23; range, $18-55$; 6 female).

\section{TMS}

Each rTMS session consisted of 900 biphasic stimuli produced by a Magstim Rapid stimulator and delivered with a frequency of $1 \mathrm{~Hz}$ over 15 min. One of two identical, standard 70-mm-diameter figure-of-eight coils was centered over the stimulation site and maintained in this position by a coil holder; the participant's head was restrained by a chin rest. The stimulation site was mapped in each subject in relation to the "motor hotspot" of the left hemisphere, which is the scalp projection of the primary motor cortex (MC). The motor hotspot was defined as the point of maximum evoked motor response in the relaxed first dorsal interosseus (FDI) muscle of the right hand. The anterior parietal cortex (APC) site of stimulation was located at $3 \mathrm{~cm}$ posterior to the motor hotspot, measured on a line oriented at $45^{\circ}$ from the sagittal plane and perpendicular on the central sulcus. The control site of stimulation was located at the motor hotspot. Stimulation intensity was set at $110 \%$ of resting motor threshold of the right FDI muscle. To identify the threshold, the subjects were asked to rest the right hand on the table with the fingers slightly spread. The resting motor threshold was then defined as the lowest intensity that reliably elicited a visible twitch in the FDI muscle when the stimulation was given over the motor hotspot. During rTMS, the coil was positioned tangential to the scalp with the long axis of the figure-of-eight coil oriented at $45^{\circ}$ to the parasagittal plane. The current flow of the initial rising phase of the biphasic pulse in the TMS coil induced a current flowing from posterior to anterior in the brain. During each session, the active coil was exchanged for the spare coil after 4 and 11 min of rTMS to avoid overheating. All subjects were tested on the tasks within a period 4-8 $\mathrm{min}$ after the cessation of the rTMS train.

\section{Design}

Each participant underwent two sessions, with rTMS at either the target or the control site. The order of the sessions was randomized across participants and scheduled on separate days. During each session, the participant was tested in their report of visually perceived straight-ahead position before (pre-TMS) and after (post-TMS) $15 \mathrm{~min}$ of $1 \mathrm{~Hz}$ rTMS.

To prevent any potential visual recalibration of eye position at perceived straight ahead (experiment 1 ) or of body midline (experiments 2 and 3), the participants were instructed to keep their eyes closed while receiving rTMS, during transfer to the testing station, and until the end of the post-TMS data collection (experiments 2 and 3 ) or until the postTMS data collection required monocular vision (experiment 1 ).

\section{Tasks}

Experiment 1: visual localization. To test for an effect of anterior parietal rTMS on perceived eye position, we measured the error in reporting the visually perceived straight-ahead position, a task sensitive to changes in eye position (Gauthier et al., 1990). The task was performed in darkness to avoid background cues and using monocular vision to rule out the detection of straight ahead based on the symmetry of the target projection on the two retinas.

The participants sat in a completely dark room in front of an array of 48 light-emitting diodes (LEDs) at $2^{\circ}$ visual angle separation. The head was fixed using a chin rest and cheek pads. Before placing the cheek pads, the experimenter checked the alignment of the body midline with the chin rest as well as the alignment of the head with the center of the LED array using the symmetry of the projection from two laser pointers placed above the ears. At the beginning of each trial, a random LED was lit, and the participants verbally instructed the experimenter to move the LED toward the left or right until the participant perceived it to be directly in front of their nose. The position of the reported LED was saved and a new trial started. LED location at perceived straight ahead was transformed into degrees of visual angle and averaged across trials within each condition.

After completing five trials, and to test whether the shift in the perceived straight ahead was related to a change in eye proprioception, we then applied a brief passive eye displacement on the closed right eye before each of another five trials and measured its effect on the TMSinduced error. The participant placed their left index finger on the right eyelid at a superior and lateral position and pushed briefly (for $\sim 1 \mathrm{~s}$ or less) the closed eye from right to left before each trial, displacing the eye leftward. After the end of the push, the subject opened the eye and started the task. Under these conditions, we do not know whether the passive displacement was fully corrected at the beginning of the trial or not. To compute eye position based on the efferent copy of the motor command, the brain needs information about this initial passive eye displacement (or the actual eye position at the beginning of the trial if a corrective movement in the opposite direction was made). In the dark, this information can only be available through eye muscle proprioception. With intact proprioception, this initial estimate of eye position would be accurate, and therefore the perceived eye position at the end of the trial would also be accurate. But with reduced proprioception, the leftward passive rotation of the eye at the beginning of the trial would be underestimated. So the final eye position based on the misjudged initial eye position and on efferent copy of the motor command would be biased rightward, in the opposite direction of the displacement. We predicted that this will add to the effect of rTMS, so that after a passive displacement and rTMS, both the final position estimate based on proprioception alone and the final position estimate based on the efferent copy and initial passive displacement would be biased toward the right.

Before the experiment, participants practiced pushing their right eye during normal binocular vision and increased the force until they produced double vision, a sign that indicated passive displacement of one eye. They were instructed to reproduce the same force when pushing the eye through the closed eyelid. After each brief push to the eye, the participants opened the eye to perform the perceived straight-ahead task.

Each session consisted of three blocks with five trials each. The blocks were to report visual straight ahead using right monocular vision, left monocular vision, and right monocular vision after passive eye displacement. The order of the first two blocks was randomized across participants. The block with a push to the eye was always presented at the end of the session to avoid interference from the passive eye displacement on the performance in the other two blocks.

Immediately after the 15 min rTMS was applied, the subject was moved to a dark room with their eyes closed, seated, and then head fixed to align head and body midline to the center of the LED array. Each test session including three blocks of five trials took $<4 \mathrm{~min}$ to complete and started no more than 4 min after the end of the TMS train.

Experiment 2: nonvisual localization using head pointing. Participants sat with the eyes closed and were asked to rotate their head so that the nose pointed straight ahead. A position-tracking system (Fastrak; Polhemus) was aligned with the $x$-axis parallel with the participant's shoulders and the origin placed at $\sim 20 \mathrm{~cm}$ from the participant's right shoulder. To record the lateral deviation of the head on trunk position, sensors were attached to the participant's trunk (just under the sternal notch) and to the forehead (just above the nose), on top of two stickers. For safety reasons, the sensors were removed before rTMS and reattached on top of the stickers after the last TMS pulse.

Each data acquisition block consisted of five trials. At the start of each trial, the head was passively rotated to one of five positions (no rotation and small-amplitude and large-amplitude passive rotation to the left or right) in a predefined pseudorandom order. The participant then moved 
back to their perceived straight-ahead position. Sensor position was averaged over measurements taken for an interval of $1 \mathrm{~s}$ at $60 \mathrm{~Hz}$. The position deviation of the head on the shoulders was calculated relative to the trunk by subtracting trunk sensor from head sensor position.

To remove any potential difference in the position of the stickers across the two TMS sessions, within each session post-TMS data were normalized by subtracting pre-TMS values. Because of instrument failure, we were not able to record sensor rotation. Instead, we used change in forehead marker position on the lateral $(x)$ and sagittal $(y)$ axes to estimate head rotation, assuming a $10 \mathrm{~cm}$ distance between the forehead and the axis of rotation. The angle of rotation was calculated as arcsin $(x / 10)$, where $x$ is the lateral displacement of the sensors, e.g., $x=$ $\left(x_{\text {postTMS,head }}-x_{\text {postTMS,trunk }}\right)-\left(x_{\text {preTMS,head }}-x_{\text {preTMS,trunk }}\right)$.

With a position resolution and positional static accuracy quoted at $<0.1 \mathrm{~cm}$, the Polhemus would have been sensitive enough to detect angular changes of $<0.57^{\circ}$.

Experiment 3: nonvisual localization using finger pointing. Participants sat with the eyes closed at a table with the head on a chin rest aligned with the body midline. A ruler with a millimetric scale was placed on the table at $50 \mathrm{~cm}$ in front of them, parallel with the shoulders and with the middle of the ruler corresponding to the body midline. A black dot was drawn on the nail of the left index finger at the beginning of each session. Each trial started with the experimenter placing the participant's finger at one of six points on the ruler in predefined random order. The points were located at 3,6 , and $11 \mathrm{~cm}$ to left or right of the body midline. The participants were instructed to slide the finger along the ruler until they felt the finger was in front of their body midline. The experimenter noted the position of the black dot on the ruler in this position. Each data acquisition block consisted of six trials, presented in the same order before and after rTMS.

\section{Results}

\section{Experiment 1}

Before TMS, participants were accurate in locating visual targets relative to the body midline. The pre-TMS error in target location at visually perceived straight ahead was $+0.85 \pm 2.68^{\circ}$ visual angle (mean \pm deviation; positive values to the right) for the right eye and $+0.17 \pm 2.34$ for the left eye, with no statistically significant difference between them (paired-samples $t$ test; $N=8$ participants; $p>0.4$ ).

After rTMS over the left APC, the visually perceived straight ahead was shifted toward the left by $-3.65 \pm 2.28^{\circ}$ (mean \pm deviation of the post-TMS minus pre-TMS difference) for the right eye and $-5.05 \pm 3.68$ for the left eye. The effect of APCrTMS was significant for both right eye ( $p=0.003$, pre-TMS vs post-TMS, paired-samples $t$ test) and left eye ( $p=0.006)$. After rTMS over the left motor cortex, the change in perceived straight ahead was at $+0.7 \pm 2.37^{\circ}$ visual angle for the right eye and $-0.55 \pm 2.51^{\circ}$ for the left eye, with no significant difference between pre- and post-TMS ( $p>0.4)$. The interaction between area (APC or MC) and TMS (pre-TMS or post-TMS) was statistically significant for both the right eye $(F=13.65 ; p=0.008)$ and for the left eye ( $F=10.30 ; p=0.015)$ (Fig. 1). This suggests that the error in the perception of straight ahead after APC-rTMS cannot be explained by an unspecific effect of rTMS or by a direct effect on the eye muscles or on their motor representation.

Brief passive displacement of the right eye before each trial did not affect the perception of straight ahead before TMS. Thus, the error after a push to the eye was $+0.32 \pm 2.91^{\circ}$, not significantly different from the error in the absence of a push $\left(+0.85 \pm 2.68^{\circ}\right.$; paired-samples $t$ test, $p>0.3$ ). After TMS over the APC, passive eye movement reduced the shift in the perceived straight ahead. Thus, with passive eye movement the shift in straight ahead was $-1.95 \pm 2.37^{\circ}$ to the left, significantly different from the shift in straight ahead in the absence of a passive eye movement $(-3.65 \pm 2.28$; paired-samples $t$ test, $p=0.047)$. After the motor cortex rTMS, the shift in straight ahead after a push to the right

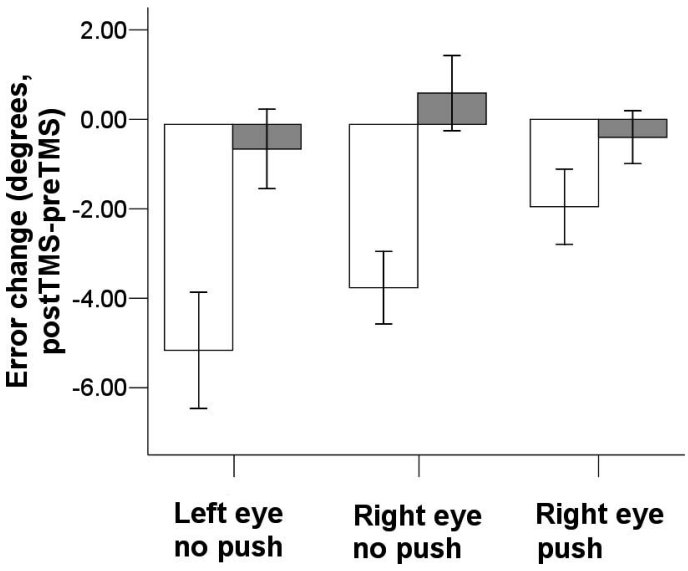

Figure 1. rTMS induced change in the perception of straight ahead (mean $\pm \mathrm{SE}$ ) in left and right monocular vision (open bars, anterior parietal cortex; filled bars, motor cortex; push, passive eye movement in medial direction applied to the closed right eye before the task).

eye was $-0.4 \pm 1.66^{\circ}$, not significantly different from the shift in straight ahead in the absence of a push $(p>0.2)$. The interaction between area (APC or MC), passive eye movement (present or absent), and TMS (pre-TMS or post-TMS) was statistically significant $(F=6.23 ; p=0.041)$ (Fig. 1$)$.

\section{Experiment 2}

rTMS over left APC did not change the nonvisual perception of body midline when subjects indicated this position by rotating the head on the trunk. Before TMS, at perceived straight ahead, the forehead sensor was located at $-1.54 \pm 1.37^{\circ}$ to the left of the trunk sensor. After rTMS, the shift in straight ahead was $-0.41 \pm$ $1.41^{\circ}$ to the left, whereas for rTMS over MC it was $-0.64 \pm 1.24^{\circ}$ to the left, with no statistically significant difference between them (paired-samples $t$ test, $p>0.7$ ). The $95 \%$ confidence interval for the difference between these two means was $[-1.2$ to $+1.7]^{\circ}$. So it is highly unlikely that the post-TMS difference in the visual perception of straight ahead, which at $>4^{\circ}$ is well outside this confidence interval (experiment 1, post-APC-rTMS minus pre-APC-rTMS vs post-MC-rTMS minus pre-MC-rTMS), merely reflects a change in perceived head position.

\section{Experiment 3}

Anterior parietal rTMS did not change the perceived body midline when participants indicated this direction by pointing with left index finger. Before TMS, the perceived body midline was at $+0.86 \pm 2.28^{\circ}$ to the right of the true body midline. The shift in perceived body midline was $+0.36 \pm 3.10^{\circ}$ to the right for APCrTMS and $+1.0 \pm 3.94^{\circ}$ to the right for MC rTMS with no significant difference between them (paired-samples $t$ test, $p>0.6$ ). The $95 \%$ confidence interval for the difference in mean was $[-3.66 \text { to }+3.31]^{\circ}$ from body midline. As before, the post-TMS difference in the visual perception of straight ahead between APC and $\mathrm{MC}$ found at experiment 1 falls outside this confidence interval.

\section{Discussion}

We investigated whether the human anterior parietal cortex codes eye position by testing for an error in visual localization after rTMS of the left APC. rTMS over the left APC increased the error in visual localization relative to the midsagittal plane by shifting the visually perceived straight ahead to the left. We interpret this shift as an error in perceived eye position. 


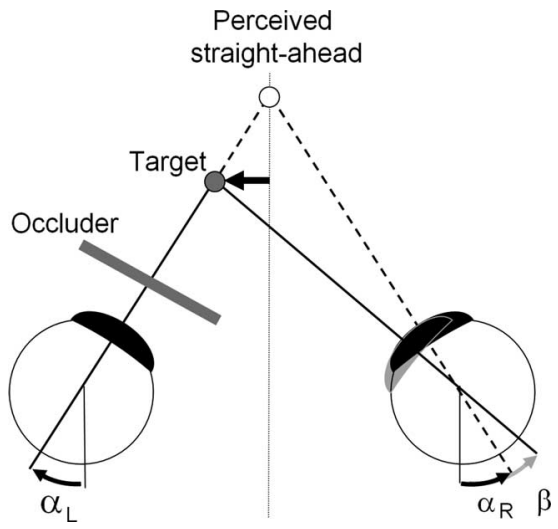

Figure 2. An underestimation $(\beta)$ of the rotation of the right eye at fixation shifts the perceived location of the target to the right and thus shifts the reported position of a target perceived as straight ahead to the left (black arrow). A mechanism for detecting perceived straight ahead in monocular vision is assumed based on equaling the felt angle of rotation of the two eyes $\left(\alpha_{R}\right.$ and $\left.\alpha_{L}\right)$; the actual angle of rotation of the right eye is $\left(\alpha_{R}+\beta\right)$. An underestimated passive rotation of the right eye toward the left would also lead to a leftward shift of perceived straight ahead.

The task in this experiment required a spatial match between a single visual target seen with monocular vision in an otherwise dark room to the midsagittal plane of the body. Therefore, an alternative explanation of our findings is that an error in perceiving the body midline after APC-rTMS may be responsible for this shift in the perception of straight ahead. However, the findings of experiments 2 and 3 ruled out this alternative explanation: in the absence of vision, the perception of body midline after APCrTMS was accurate when tested with either finger or with head pointing. These findings rule out an error in the body schema or in the comparison between effector position and the body schema as an explanation of error in visual localization relative to body.

Detection of straight ahead in monocular vision takes into consideration eye position information from both eyes regardless of whether one eye is occluded or not (Gauthier et al., 1990). For a target $57 \mathrm{~cm}$ from the eyes and placed at either -3.65 or -5.05 $\mathrm{cm}$ to the left of the midsagittal plane, and assuming an interocular separation of $8 \mathrm{~cm}$, at fixation the right eye would be rotated medial to the sagittal plane by -7.65 or $-9.05^{\circ}$, respectively. The left eye would be rotated by $+0.35^{\circ}$ medial and $-1.05^{\circ}$ temporal, respectively. If we make the assumption that the reported straight-ahead position was achieved by matching the felt position of left and right eyes, then the actual rotation of the right eye is underestimated and is felt to be nearly parallel with the sagittal plane. Thus, a shift of the perceived straight ahead toward the left reflects an underestimated leftward rotation of the right eye at fixation (Fig. 2).

Because the rTMS can induce changes in neural activity that are not restricted to the area directly underneath the coil, we cannot rule out that the effect of rTMS on eye position representation occurs via connections from the anterior parietal cortex to other brain areas (e.g., posterior parietal cortex). However, the current results match the single-cell recording data in the monkey, in which anterior parietal neurons code an eye position signal that increases monotonically with eye eccentricity in the orbit (Wang et al., 2007). Cortical depression caused by rTMS would be expected to reduce the slope of this relationship, so that eye eccentricity would be underreported by this area. This fits with the present results and suggests that the anterior parietal cortex itself may be responsible for the effect.
The size of the shift to the left in the perceived straight ahead induced by anterior parietal rTMS was modulated by passive eye movement. This finding strengthens the association between the effect of APC-rTMS and an accurate perception of eye position. With an initial passive leftward eye displacement and reduced proprioception, we expected that the final eye position estimate, based on a correct efferent copy of the motor command and an underestimated initial displacement, to be to the right of the true eye position. This would have caused a rightward shift in the perceived location of the target and a leftward shift in the perceived straight ahead (Fig. 2). This was expected to increase even further the leftward shift in perceived straight ahead caused by APC-rTMS alone. However, our result showed that the opposite was the case: leftward passive eye movement reduced rather than increased the leftward shift in the perceived straight ahead caused by APC-rTMS. Therefore, although the presence of an interaction between passive eye displacement and TMS suggests that an orbital proprioceptive signal is altered by APC-rTMS, an assumption of an inaccurately perceived initial position alone cannot explain this effect. We cannot yet explain why a left displacement applied to the eye decreased the leftward rTMS shift in the perception of straight ahead. We speculate that it may reflect a more complex interplay between proprioception and the efferent copy of the motor command in the estimate of initial eye position, e.g., the calibration of the efferent copy of the motor command by eye proprioception (Steinbach, 1986).

Another possibility is simply that our tests of passive eye displacement on perceived straight ahead were conducted later in the post-TMS period, and the effect of TMS may have diminished by that time. The exact duration of the depression caused by 15 min of $1 \mathrm{~Hz}$ TMS is not known; data from rTMS applied to motor cortex (Muellbacher et al., 2000; Touge et al., 2001) suggest the effect might last 10-20 min, but as a conservative rule of thumb, an interval of $50 \%$ of stimulus duration $(7.5 \mathrm{~min})$ is often assumed (Robertson et al., 2003). To exclude such a timedependent effect, we reanalyzed our subject-specific data into two groups that differed by the time interval between the right eye-no push and -push conditions. Half the participants (group 1) performed the conditions in the order right eye-no push, left eye-no push, right eye-push, whereas for the other half (group 2) this order was left eye-no push, right eye-no push, right eye-push. We reasoned that if a reduction in the effect of rTMS with time was responsible for the reduction in straight-ahead error from the right eye-no push condition to the right eye-push condition, then this reduction should be larger in group 1, in which these two conditions were separated by a longer time interval than in group 2. This was not the case. The reduction (push - no push) for group 1 was $1.6 \pm 2.2^{\circ}$, whereas the reduction for group 2 was $1.8 \pm 0.9^{\circ}$, which argues against a time effect as an explanation of our findings. Moreover, we also tested for a time effect in the first two blocks, in which the order of left and right eye condition was balanced across subjects and failed to find a time-dependent effect in the shift of the perceived straight ahead $(2 \times 2$ ANOVA, with an insignificant interaction between TMS site and session order $\left(F_{(1,7)}=0.01 ; p>0.9\right)$. The main effect of TMS site was significant $(p=0.007)$. Therefore we think it is unlikely that the reduction in leftward shift of perceived straight ahead tested after passive eye displacement can be explained only by the decay of the effect of rTMS over time.

We conclude that the human anterior parietal cortex encodes eye position and that this eye position signal has a proprioceptive component. The identification of a brain area in humans that represents eye position and the finding that rTMS can interfere 
with this signal open the possibility of studying the influence of perceived eye position in visual attention and visuomotor behavior in healthy and patient populations.

\section{References}

Andersen RA, Mountcastle VB (1983) The Influence of the angle of gaze upon the excitability of the light-sensitive neurons of the posterior parietal cortex. J Neurosci 3:532-548.

Balslev D, Christensen LO, Lee JH, Law I, Paulson OB, Miall RC (2004) Enhanced accuracy in novel mirror drawing after repetitive transcranial magnetic stimulation-induced proprioceptive deafferentation. J Neurosci 24:9698-9702.

Barker AT (1999) The history and basic principles of magnetic nerve stimulation. Electroencephalogr Clin Neurophysiol Suppl 51:3-21.

Craighero L, Nascimben M, Fadiga L (2004) Eye position affects orienting of visuospatial attention. Curr Biol 14:331-333.

DaSilva AFM, Becerra L, Makris N, Strassman AM, Gonzalez RG, Geatrakis N, Borsook D (2002) Somatotopic activation in the human trigeminal pain pathway. J Neurosci 22:8183-8192.

Donaldson IM (2000) The functions of the proprioceptors of the eye muscles. Philos Trans R Soc Lond B Biol Sci 355:1685-1754.

Gauthier GM, Nommay D, Vercher JL (1990) The role of ocular muscle proprioception in visual localization of targets. Science 249:58-61.

Han Y, Lennerstrand G (1999) Changes of visual localization induced by eye and neck muscle vibration in normal and strabismic subjects. Graefes Arch Clin Exp Ophthalmol 237:815-823.

Lewis RF, Gaymard BM, Tamargo RJ (1998) Efference copy provides the eye position information required for visually guided reaching. J Neurophysiol 80:1605-1608.

Muellbacher W, Ziemann U, Boroojerdi B, Hallett M (2000) Effects of lowfrequency transcranial magnetic stimulation on motor excitability and basic motor behavior. Clin Neurophysiol 111:1002-1007.

Nguyen BT, Tran TD, Hoshiyama M, Inui K, Kakigi R (2004) Face representation in the human primary somatosensory cortex. Neurosci Res 50:227-232.

Pavani F, Làdavas E, Driver J (2005) Gaze direction modulates auditory spatial deficits in stroke patients with neglect. Cortex 41:181-188.

Robertson EM, Théoret H, Pascual-Leone A (2003) Studies in cognition: the problems solved and created by transcranial magnetic stimulation. J Cogn Neurosci 15:948-960.

Steinbach MJ (1986) Inflow as a long-term calibrator of eye position in humans. Acta Psychol (Amst) 63:297-306.

Touge T, Gerschlager W, Brown P, Rothwell JC (2001) Are the after-effects of low-frequency rTMS on motor cortex excitability due to changes in the efficacy of cortical synapses? Clin Neurophysiol 112:2138-2145.

Wang X, Zhang M, Cohen IS, Goldberg ME (2007) The proprioceptive representation of eye position in monkey primary somatosensory cortex. Nat Neurosci 10:640-646. 